\title{
Educação em saúde para prevenção e controle da hanseníase voltada para agentes comunitários de saúde: relato de experiência
}

\author{
Health education for the prevention and control of \\ hanseniasis returned to community health agents: \\ experience report
}

\author{
Jamylle Silva Campos', Aline Silva Castro', Shaumin Vasconcelos Wu', \\ Raphaely Cristiny Sanches Progênio', João Paulo Menezes Lima', \\ Biatriz Araújo Cardoso', Angélica Homobono Nobre' \\ 'Universidade do Estado do Pará, Belém (PA) Brasil.
}

\section{RESUMO}

Contextualização: No Brasil, a hanseníase é considerada uma doença endêmica. Dessa forma, programas de controle da hanseníase na rede básica de saúde são considerados como estratégia para o controle da doença e melhoria na qualidade do atendimento prestado a pessoa com hanseníase, facilitando o acesso ao tratamento, a prevenção de incapacidades, a diminuição do estigma e da exclusão social. Assim, torna-se fundamental o papel da atenção primária na erradicação da doença. Descrição da experiência: O objetivo principal foi relatar a experiência vivenciada na realização da educação em saúde voltada para os Agentes Comunitários de Saúde (ACS), para que pudessem realizar busca ativa, identificar precocemente os casos e orientar a população quanto a prevenção e controle da a doença. Para isso, foi realizada uma oficina que contou com a participação de 55 ACS do município de Bujaru (PA). Na oficina foram abordados temas como: histórico da hanseníase no Brasil e no mundo, fisiopatologia da hanseníase, sinais e sintomas, formas de diagnóstico, tratamento, autocuidado, prevenção de incapacidades, papel do ACS na prevenção e controle da doença. Utilizou-se metodologia pedagógica como rodas de conversa e gincanas educativas, através de dinâmicas de verdadeiro ou falso, "perguntas na caixa" e um jogo de tabuleiro, para abordar os assuntos elencados. Considerações finais: A oficina contribuiu para enriquecer o conhecimento dos participantes acerca dos aspectos da hanseníase. Mostrando que a educação em saúde é uma ferramenta fundamental para a interação entre os saberes envolvidos no processo saúde-doença, na capacitação dos gestores do sistema de saúde, na prevenção de doenças, orientações e promoção de saúde.

\section{COMO CITAR ESTE ARTIGO}

Campos JS, Castro AS, Wu SV, et al. Educação em saúde para prevenção e controle da hanseniase voltada para e controle da hanseniase voltada agentes comunitários de saúde: relato de experiência. Interdisciplinary Journal of Health Education. 2020 Jan-Jun;5(1):40-45. https://doi. org/10.4322/ijhe.2020.005

\section{CORRESPONDÊNCIA}

Angélica Homobono Nobre Departamento de Ciências do Movimento Humano, Universidade do Estado do Pará

Travessa Perebebuí, 2623, Bairro do Marco, CEP 66095-662, Belém (PA) Brasil

angelica.homobono@gmail.com

\section{FONTE DE FINANCIAMENTO}

Financiamento próprio.

\section{CONFLITO DE INTERESSE}

Os autores declararam não

haver conflitos de interesse.

O estudo foi realizado na

Secretaria Municipal de Saúde

de Bujarú, Bujarú (PA), Brasil

PALAVRAS-CHAVE: Relações comunidade-instituição. Capacitação em serviço. Atenção Primária à Saúde.

\begin{abstract}
Contextualization: In Brazil, leprosy is considered an endemic disease. Thus, leprosy control programs in the basic health network are considered as a strategy for controlling control the disease and improving the quality of care provided to the person with leprosy, facilitating access to treatment, prevention of disabilities, reduction of stigma and social exclusion. So, the role of primary care in the eradication of the disease becomes fundamental. Description of the experience: The main objective was to report on the experience of health education for the Community Health Agents (ACS), so that they could perform an active search, identify the cases early and guide the population on the prevention and control the disease. For this, a workshop was held with the participation of 55 ACS from the municipality of Bujaru (PA). The workshop addressed topics such as: history of leprosy in Brazil and in the world, pathophysiology of leprosy, signs and symptoms, forms of diagnosis, treatment, self-care, disability prevention and the role of ACS in the prevention and control of disease. Pedagogical methodology was used as conversation wheels and educational scoundrels, through true or false dynamics, "questions in the box" and a board game, to address the issues listed. Final considerations: The workshop contributed to enrich participants' knowledge about the aspects of leprosy. The results showed that health education is a fundamental tool for the interaction between the knowledge involved in the health disease process, in the training of health system managers, in disease prevention, guidance and health promotion
\end{abstract}

KEYWORDS: Community-institutional relations. Training in service. Primary Health Care. 


\section{Contextualização}

A hanseníase é uma doença endêmica, sendo o Brasil o país com maior número de casos na América Latina e o segundo no mundo. Sua distribuição é heterogênea nas regiões brasileiras, destaca-se a região norte e nordeste pelo elevado índice da doença entre crianças e adolescentes ${ }^{1,2}$.

Em razão disso, os programas de controle da hanseníase na rede básica de saúde são considerados, atualmente, como estratégia para eliminação da doença, para o diagnóstico precoce e melhoria na qualidade do atendimento prestado. Isso facilita o acesso ao tratamento, a prevenção de incapacidades, a diminuição do estigma e da exclusão social ${ }^{3}$. Sendo assim, a atenção primária possui um papel relevante na erradicação da doença ${ }^{4}$.

A ampliação da rede de atenção à saúde e preparação dos profissionais de saúde para o atendimento qualificado e humanizado são desafios para alcançar um melhor controle e prevenção da hanseníase no país. Nesse contexto, destaca-se a educação em saúde como ferramenta para resoluções favoráveis para formação continuada dos demais profissionais, quanto para interação de práticas e saberes em saúde que permitirão uma real aproximação das demandas da população ${ }^{5}$.

O preconceito pode permear a vida do paciente acometido por hanseníase e, portanto, as dificuldades encontradas para inserção no meio social estão diretamente relacionadas à adoção de práticas inclusivas pelos serviços de saúde. Desse modo, as políticas educativas sobre a doença contribuem para a diminuição do estigma da hanseníase, diminuindo assim a prevalência da doença ${ }^{6}$.

Os agentes comunitários de saúde (ACS) são importantes no combate da hanseníase, pois estão, diretamente, ligados à comunidade, promovem ações educativas e realizam a busca ativa ${ }^{7}$. Assim, a educação em saúde pode ser vista como uma possibilidade para enfrentar os desafios da saúde, com destaque para a hanseníase e para atuação desse profissional, visto necessidade da superação do olhar estigmatizante sobre a doença e do diagnóstico precoce ${ }^{8}$.

\section{Descrição da experiência}

Trata-se de um relato da experiência vivenciado em uma oficina educativa em saúde, realizada no município de Bujaru, estado do Pará, o qual pertence à mesorregião metropolitana de Belém. Todas as ações foram executadas no espaço da Escola Estadual de Ensino Dom Mário de Miranda Vilas Boas.

A proposta da atividade foi aprovada em edital público para extensão universitária, por meio do programa Campus Avançado, sendo celebrada parceria entre a Universidade do Estado do Pará (UEPA), que disponibilizou os recursos materiais e humanos para execução do trabalho, e a Secretaria de Saúde da Prefeitura Municipal de Bujaru, atuando por meio da alocação e hospedagem dos alunos, além da logística e mobilização dos participantes. As atividades foram realizadas na modalidade de oficina de capacitação que ocorreu no período de 25 a 29 de julho de 2016, nos turnos da manhã e da tarde.

O objetivo principal do trabalho foi capacitar os profissionais da rede pública municipal de saúde que atuassem na atenção primária, a fim de que esses pudessem ser capazes de identificar possíveis casos de hanseníase, orientar a população sobre as formas de prevenção e controle da doença. Para isso, optou-se por realizar a capacitação dos ACS, visto que são os profissionais de maior contato com a população, principalmente aquela que residiam em comunidades distantes do centro da cidade. Assim, mobilizados pela secretaria municipal de saúde, os 55 ACS do município foram convocados para participar da oficina.

A metodologia empregada dividiu o processo de capacitação em cinco etapas: treinamento dos discentes envolvidos no projeto; investigação do conhecimento 
dos participantes; capacitação dos ACS; avaliação da percepção dos participantes e encerramento; avaliação e percepção da equipe de trabalho.

Após a aprovação da atividade de extensão, o coordenador do projeto realizou a capacitação dos discentes, onde foram instigados a aprofundar seus conhecimentos sobre a temática e sobre formas pedagógicas de educação para profissionais de saúde. Também neste momento, foi formulada uma lista de frequência, para ter controle do número de participantes diários, e um questionário com perguntas sobre tempo de atuação como ACS, nível de escolaridade, e sobre a temática, para investigação do conhecimento e avaliação da percepção dos participantes, assim possuindo perguntas objetivas e discussivas. O questionário foi aplicado antes e após a oficina.

No primeiro da oficina, foi realizada a apresentação da equipe e da proposta de trabalho, seguido da aplicação do questionário para análise do conhecimento prévio e diálogo sobre a percepção dos ACS em relação ao cenário da hanseníase vividos pelos mesmos no município e como realizavam a abordagem do tema dentro das visitas domiciliares. Além disso, durante a conversa, os discentes introduziram alguns conceitos e questões relacionadas à doença para que pudessem traçar um perfil dos participantes e dos conhecimentos prévios que possuíam, para que assim pudessem conduzir as ações educativas de forma mais eficiente.

Nos dias seguintes deu-se início as atividades educativas, nas quais foram abordados os seguintes temas: histórico da hanseníase no Brasil e no mundo, políticas públicas de saúde em hanseníase, fisiopatologia da hanseníase, formas clínicas, sinais e sintomas, formas de diagnóstico e diagnóstico diferencial, tratamento, autocuidado, prevenção de incapacidades, papel dos ACS na prevenção da doença e seus agravos.

Para a abordagem dos assuntos descritos, adotou-se como metodologia pedagógica da oficina a execução de aulas expositivas, rodas de conversa com pequenos grupos e gincanas educativas, como dinâmicas de verdadeiro ou falso, "perguntas na caixa" e jogo de tabuleiro. Importante ressaltar, que as perguntas realizadas durante a dinâmica eram feitas com base no conteúdo ministrado no dia e nas próprias dúvidas dos profissionais, identificadas no questionário e ao decorrer das aulas.

A dinâmica de verdadeiro ou falso foi realizada por meio da apresentação de diversas afirmativas, formuladas pela equipe de trabalho, na qual os participantes deveriam levantar a placa em formato de "V" para aquelas que julgassem estar corretas e placa em formato de " $\mathrm{F}$ " para as que acreditassem estar erradas. A partir disso, era revelado se a frase era verdadeira ou falsa e era realizada uma breve revisão sobre o assunto e as devidas correções.

Também foi realizada a dinâmica de "perguntas na caixa", onde foram feitas perguntas escritas em uma tira de papel e colocadas em uma caixa. Os participantes foram organizados em filas, sentados em suas cadeiras, pelas quais a caixa passava após sua entrega para um deles. Posteriormente os alunos colocavam uma música para ser executada enquanto os participantes passavam a caixa para o colega do lado e assim, sucessivamente, até que o som fosse pausado. A partir disso, aquele que tivesse com a caixa em mãos no momento da pausa, deveria retirar uma questão e respondê-la. A dinâmica seguia até que todas as perguntas fossem respondidas.

Outra dinâmica elaborada pela equipe foi a gincana do tabuleiro (Figura 1), a qual gerou extremo interesse e participação dos ACS, sendo realizado no pátio da escola. Ocorreu a confecção de um tabuleiro composto de 16 casas, possuindo linha de partida e de chegada, um dado com 6 faces e pinos que eram os próprios integrantes do jogo. Os participantes da oficina foram divididos em equipes, identificadas através das cores amarelo, azul, verde e branco. Os mesmos deveriam eleger um representante para tornar-se o pino do jogo e um para jogar o dado, os demais iriam contribuir auxiliando o representante na formulação das respostas.

No tabuleiro cada casa possuía cerca de cinco perguntas, para caso as equipes ocupassem a mesma casa, sendo feita ao mesmo tempo ou na jogada seguinte. 


\section{ijhe}

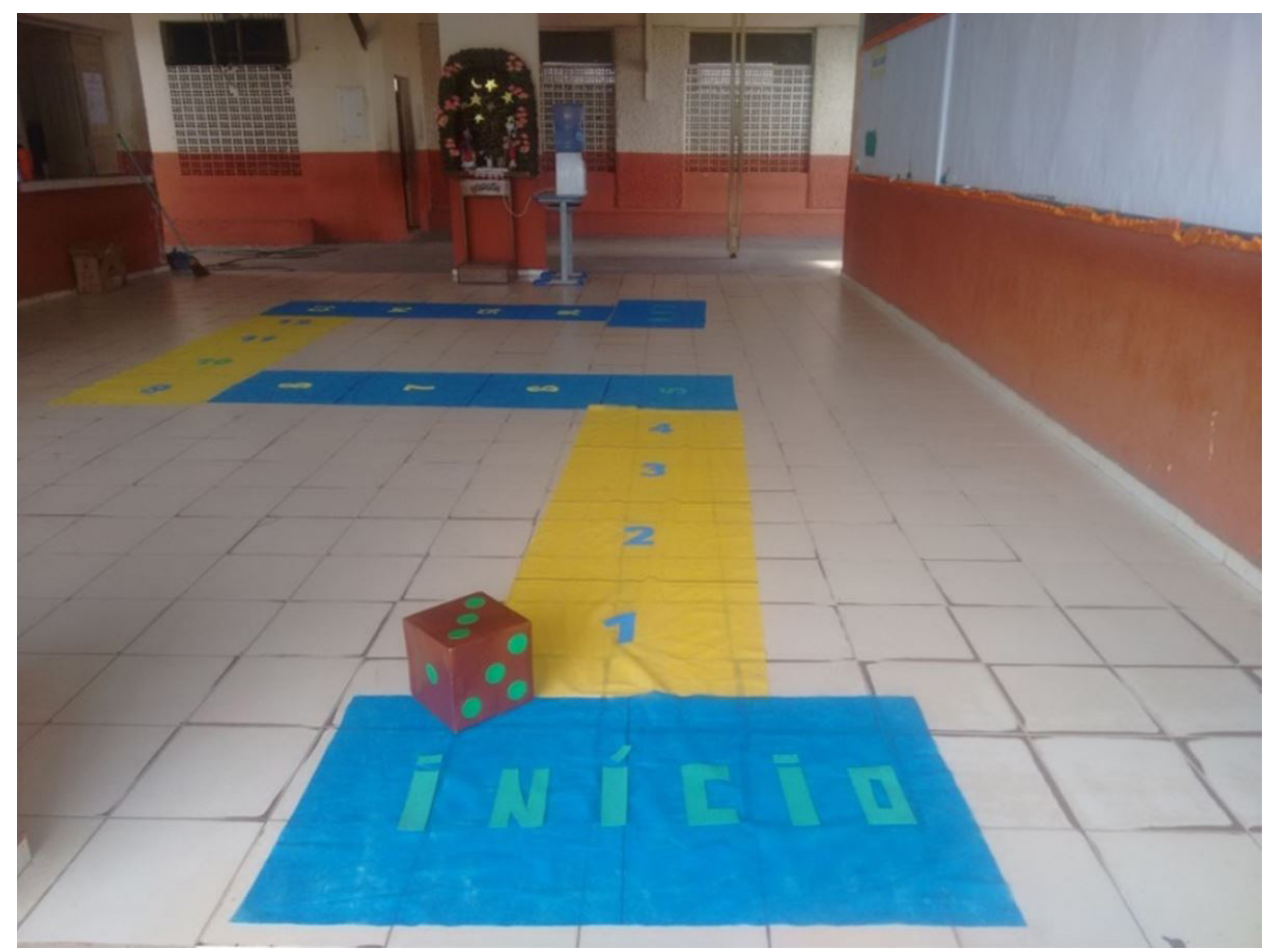

Figura 1. Jogo de tabuleiro.

Fonte: arquivo pessoal.

As perguntas possuíam o mesmo nível de dificuldade e conforme os jogadores avançavam o nível de dificuldade aumentava.

No último dia da oficina, após a gincana, os participantes foram convidados a voltar a sala de aula para a roda de conversa onde puderam expressar sua visão sobre a capacitação, o que foi absorvido dos cinco dias, suas principais dificuldades e como o projeto contribuirá para as ações futuras na comunidade.

Notoriamente, mesmo que com uma avaliação subjetiva, os ACS conseguiram alcançar o objetivo do trabalho e demonstraram grande interesse em participar novamente de mais capacitações. Por fim, foi realizada uma cerimônia de encerramento, momento em que foi entregue o certificado de participação dos ACS na oficina.

\section{Resultados e impactos}

Houve a participação de 55 agentes, população total de ACS da cidade. Sendo que a frequência desses variou de 48 a 52 agentes, segundo a lista de presença aplicada em cada dia.

A oficina possibilitou aos participantes enriquecer seus conhecimentos acerca dos diversos aspectos da hanseníase, visto que, durante a execução das atividades ocorreram momentos de discussão a respeito do tema, permitindo o esclarecimento de questões que envolviam a atuação desses profissionais dentro da comunidade.

Além disso, a utilização de uma abordagem diferenciada e dinâmica incentivou maior adesão dos profissionais à proposta do estudo, bem como maior interesse pelas aulas. Durante os cinco dias de oficina houve uma grande participação dos ACS e constante demonstração de interesse sobre a temática abordada. A adesão à proposta, segundo Silva et al. (2016), ocorre quando existe uma interação de educadores e educandos, quando o assunto apresentado está relacionado ao seu cotidiano e a capacitação acontece no próprio espaço de trabalho'. 
Fomentar a educação desses profissionais é de extrema relevância para a saúde preventiva, visto que o ACS realiza um trabalho diferenciado junto à população, que englobam atividades de prevenção e vigilância em saúde, por meio de visitas domiciliares e ações de educação em saúde. O ACS é fornecedor de informações à equipe de saúde sobre a situação das famílias, principalmente aquelas em situação de risco, assumindo o papel de sujeito articulador, ressaltando a importância de sua formação ${ }^{10}$.

Assim, educação em saúde é uma ferramenta importante para capacitação do ACS, permitindo que possam estar preparados e tenham percepção para identificar os possíveis casos de hanseníase, assim como orientar de forma mais adequada os pacientes com suspeita de hanseníase e sua família.

Uma problemática observada durante os relatos na oficina foi a dificuldade do ACS em atuar como educador em saúde e utilizar sua autonomia para promoção de ações de saúde, visto que acabam se tornando dependentes dos demais profissionais da equipe para a execução desse tipo de atividade. Entretanto, vale ressaltar que os ACS foram incentivados a criar grupos de apoio para melhor acolher e informar as pessoas acometidas pela doença.

A capacitação também abordou os aspectos psicossociais da hanseníase, notou-se que muitos participantes ainda possuíam alguns preconceitos, mitos e medos em relação à doença, segundo relatos verbais e respostas discussivas presente no questionário, que por vezes influenciava na atuação com o doente e a família. Dessa forma, foi possível perceber que ainda existem muitas barreiras a serem ultrapassadas no que diz respeito aos estigmas da hanseníase, até mesmo entre os profissionais de saúde.

Para Gomes et al. ${ }^{11}$ a doença carrega um grande estigma social, ocasionando isolamento social, o que agrava a situação epidemiológica da doença no país. Assim, é importante a adoção de estratégias que possibilitem a ampliação do saber e o comprometimento dos profissionais de saúde ao combate a hanseníase, proporcionando ao paciente uma melhor qualidade de vida.

Pode-se elencar como dificuldades para execução da capacitação a distância entre o local da realização da oficina e a residência dos agentes, mas essa foi superada com o auxílio da Secretária de Saúde do município que proporcionou aos profissionais, hospedagem e alimentação durante o período da capacitação. Além disso, alguns dos ACS mostraram-se tímidos e com certa insegurança ao responder as perguntas na frente dos colegas de trabalho, entretanto, tanto a equipe de trabalho, quanto os demais participantes buscaram incentivá-los durante as respostas, buscando o máximo de aproveitamento e conforto para todos.

Ficou evidente para os pesquisadores a necessidade que o agente de saúde tem de cada vez mais aprimorar seu trabalho e o interesse em contribuir para os avanços da saúde naquela localidade. Apesar do curto período, foi possível a criação de um vínculo entre os participantes e a equipe de pesquisa, que também foi beneficiada com a oportunidade de vivenciar a realidade de saúde de uma comunidade interiorana. Dessa forma, a extensão universitária apresentou-se como uma grande ferramenta para a socialização do conhecimento e do diálogo entre o saber científico e popular ${ }^{12}$.

\section{Considerações finais}

A educação em saúde trata-se de uma ferramenta fundamental no que diz respeito à interação entre os diversos saberes envolvidos no processo saúde-doença, na capacitação dos gestores do sistema de saúde, na prevenção de doenças, orientações e promoção de saúde. Observou-se a importância de estratégias lúdicas para interação entre os participantes, envolvimento com a temática, possibilidade de troca de experiências e oportunidade de aprimorar conhecimentos de maneira lúdica. 


\section{Referências}

1. Brasil. Ministério da Saúde. Secretaria de Vigilância em Saúde. Boletim Epidemiológico. Hanseníase [Internet]. Brasília: Ministério da Saúde; 2018 [citado em 2018 Jun 25]. Disponível em: http://portalarquivos2.saude.gov.br/images/pdf/2018/ janeiro/31/2018-004-Hanseniase-publicacao.pdf

2. Ribeiro MDA, Silva JCA, Oliveira SB. Estudo epidemiológico da Hanseníase no Brasil: reflexão sobre as metas de eliminação. Rev Pan de Sal Publ. 2018;42:e42. http://dx.doi.org/10.26633/RPSP.2018.42.

3. Brasil. Ministério da Saúde. Secretaria de Vigilância em Saúde. Departamento de Vigilância de Doenças Transmissíveis. Diretrizes para vigilância, atenção e eliminação da Hanseníase como problema de Saúde Pública [Internet]. Brasília: Ministério da Saúde; 2016 [citado em 2018 Jun 25]. Disponível em: http://www.saude.pr.gov.br/arquivos/File/Manual_de_Diretrizes_ Eliminacao_Hanseniase.pdf

4. Oliveira CM, Linhares MSC, Ximenes-Neto FRG, Mendes IMVP, Kerr LRFS. Conhecimento e práticas dos agentes comunitários de saúde sobre hanseníase em um município hiperendêmico. Saud Rev. 2018;17(48):39-50. http://dx.doi. org/10.15600/2238-1244/sr.v17n48p39-50.

5. Gomes MP, Pinto MH, Nardi SMT, Paschoal VD. Hanseníase: a visão do profissional da saúde no Discurso do Sujeito Coletivo. Rev Bras Pesq Saúde. 2014;16(1):41-48. http://dx.doi.org/10.21722/rbps.v16i1.8485.

6. Sousa GS, Silva RLF, Xavier MB. Hanseníase e Atenção Primária em Saúde: uma avaliação de estrutura do programa. Saud Deb. 2017;41(112):230-42.

7. Carlos J, Ribeiro MDA, Oliveira SB. Avaliação do nível de informação sobre hanseníase dos agentes comunitários de saúde. Rev Bras Prom da Saud. 2016;29(3):364-70. http://dx.doi.org/10.5020/18061230.2016.p364.

8. Amaral MCS, Pontes AGV, Silva JV. O ensino de Educação Popular em Saúde para o SUS: experiência de articulação entre graduandos de enfermagem e Agentes Comunitários de Saúde. Interf Comun Saud Educ. 2014;18(2, suppl 2):1547-58. http:// dx.doi.org/10.1590/1807-57622013.0441.

9. Silva LAA, Schmidt SMS, Noal HC, Signor E, Gomes IEM. Avaliação da educação permanente no processo de trabalho em saúde. Trab Educ Saúde. 2016;14(3):765-81. http://dx.doi.org/10.1590/1981-7746-sol00015.

10. Maciazeki-Gomes RC, Souza CD, Baggio L, Wachs F. O trabalho do agente comunitário de saúde na perspectiva da educação popular em saúde: possibilidades e desafios. Cien Saude Colet. 2016;21(5):1637-46. http://dx.doi.org/10.1590/141381232015215.17112015. PMid:27166911.

11. Gomes MP, Pinto MH, Nardi SMT, Paschoal VDA. Hanseníase: a visão do profissional da saúde no Discurso do Sujeito Coletivo. Rev Bras Pesq Saud. 2014;16(1):41-8. http://dx.doi.org/10.21722/rbps.v16i1.8485.

12. Valdanha-Neto D, Vasconcelos VO. Educação popular e extensão universitária: ressonâncias de experiências em uma comunidade ribeirinha amazônica da região do baixo rio Madeira. Rev Cien da Educ. 2015;32:97-117.

\section{Contribuição dos autores}

Jamylle Silva Campos participou da elaboração do desenho metodológico e da supervisão do projeto, da coleta, tratamento, análise e interpretação dos dados, do levantamento bibliográfico e da redação e revisão crítica do manuscrito. Aline Silva Castro participou da elaboração do desenho metodológico e da supervisão do projeto, da coleta, tratamento, análise e interpretação dos dados, do levantamento bibliográfico e da redação e revisão crítica do manuscrito. Shaumin Vasconcelos Wu participou da elaboração do desenho metodológico e da supervisão do projeto, da análise e interpretação dos resultados, do levantamento da literatura e redação do manuscrito. Raphaely Cristiny Sanches Progênio participou da elaboração do desenho metodológico e da supervisão do projeto, da coleta, tratamento, análise e interpretação dos dados, do levantamento bibliográfico, da redação e revisão crítica do manuscrito. João Paulo Menezes Lima participou da elaboração do desenho metodológico e da supervisão do projeto, da coleta e tratamento, análise e interpretação dos dados, do levantamento bibliográfico e da redação e revisão crítica do manuscrito. Biatriz Araújo Cardoso participou da análise e interpretação dos resultados, do levantamento da literatura, redação e revisão crítica do manuscrito Angélica Homobono Nobre participou da elaboração do desenho metodológico e da supervisão do projeto, da coleta e tratamento dos dados, do levantamento biliografico e revisão crítica do manuscrito. 